\title{
Retarded response of the optical transmittance through a magnetic fluid film under switching-on/off external magnetic fields
}

\author{
S.Y. Yang ${ }^{\mathrm{a}}$, Y.T. Hsiao ${ }^{\mathrm{a}}$, Y.W. Huang ${ }^{\mathrm{a}}$, H.E. Horng ${ }^{\mathrm{a}, \mathrm{b}, *}$, \\ Chin-Yih Hong ${ }^{\mathrm{c}}$, H.C. Yang ${ }^{\mathrm{d}}$ \\ ${ }^{a}$ Institute of Electro-optical Science and Technology, National Taiwan Normal University, Taipei 116, Taiwan \\ ${ }^{\mathrm{b}}$ Department of Physics, Institute of Electro-optical Science and Technology, National Taiwan Normal University, Taipei 116, Taiwan \\ ${ }^{\mathrm{c}}$ Department of Mechanical and Automation Engineering, Da-Yeh University, Chang-Hwa 515, Taiwan \\ ${ }^{\mathrm{d}}$ Department of Physics, National Taiwan University, Taipei 106, Taiwan
}

Received 7 July 2003; received in revised form 7 January 2004

Available online 23 April 2004

\begin{abstract}
In our research, we develop the setup to observe the time-dependent response of the optical transmission through a magnetic fluid film under switching-on or -off the external magnetic field. The optical transmittance is reduced when an external magnetic field is applied perpendicularly to a magnetic fluid film. It was further found that the transmittance starts to decrease in tens of milliseconds after switching-on the external field (referred as the charging process). This retarded response of the transmittance with respect to the external of the field also occurs when the field is switched off (referred as the discharging process), with the retarding time ranging from several to tens of milliseconds, depending on field strength. In addition, the exponential decay or increase in the transmitted intensity was observed for the charging or discharging process, respectively.

(C) 2004 Published by Elsevier B.V.
\end{abstract}

PACS: 75.50.Mm; 78.20.Ci; 61.20.Lc

Keywords: Magnetic fluid; Transmittance; Retarding

\section{Introduction}

Due to potential applications, the optical phenomena of magnetic fluids subjected to an

*Corresponding author. Tel.: + 886-2-2363-1755; fax: + 8862-2362-5753.

E-mail address: phyfv001@scc.ntnu.edu.tw (H.E. Horng). external magnetic field have been recently put under extensive study. One of the areas of research done on such phenomena concentrates on the optical transmission of magnetic fluid films under an external magnetic field. When a magnetic fluid thin film is subjected to an external-pulsed magnetic field, the particles in the film rotate to align their magnetic moment to the field direction 
but are prevented from agglomerating through external control of the duration of the pulsed field within hundreds of microseconds. Because of the anisotropy in the magnetic particle shape [1,2] or the interaction between particles $[3,4]$, the magnetic fluids become optically anisotropic [5-8], and dichroism or birefringence may occur as a linearly polarized light passed normally through the thin film. By aligning the transmission axes of the linear polarizer and the analyzer so that they are perpendicular to each other, the intensity of the transmitted light increases during the application of the pulsed field. Since the dichroism or the birefringence contributes to the re-orientation of a single magnetic particle, the dynamic birefringence of a magnetic fluid under a pulsed field serves as a significant measurement with which to probe Brownian rotation of a single particle in a magnetic fluid [9-11]. It was also found that the decay time of the dynamic dichroism or birefringence is around tens of microseconds, depending on either fluid viscosity, particle size [9,11], or particle size distribution [10], etc.

When the duration of a pulsed field is extended, some of particles in the magnetic fluid film agglomerate and magnetic clusters are formed. With the magnetic clusters, the decay time of the dynamic dichroism or birefringence is larger than that in a single particle re-orientation by one order of magnitude [12]. Furthermore, the dichroism or the birefringence is enhanced with an increase in both magnetic field strength and particle concentration, and it tends to saturate at higher fields $[13,14]$. Most of the published papers are focused on discussing the relationship between the dichroism or the birefringence and the magnetic clusters when the magnetic fluid reaches equilibrium. In this work, we further investigate the real-time variation in the optical transmission through the magnetic fluid film due to formation of magnetic clusters. To do this, we develop an experimental setup to detect the time-dependent transmission for the magnetic fluid film under switching-on or off the external magnetic field. The phenomena to be observed are very important for exploring dynamic optical devices through the utilization of field-dependent transmissions of magnetic fluid film.

\section{Experimental details}

The experimental setup used is schematically illustrated in Fig. 1. An unpolarized light with a wavelength of $632.8 \mathrm{~nm}$ was emitted from a $\mathrm{He}-\mathrm{Ne}$ laser and was incident normally to the magnetic fluid film. The investigated magnetic fluid was water-based $\mathrm{Fe}_{3} \mathrm{O}_{4}$ with a concentration of $1.08 \mathrm{emu} / \mathrm{g}$ and was sealed in a square cell $11.5 \mu \mathrm{m}$ thick. A high-speed photo-detector with a response time of several pico-seconds was used to probe the time-varying intensity of the light transmitted through the film as the externally magnetic field was turned on or off. The external magnetic field was provided by a pair of solenoids and was perpendicular to the plane of the film. The probed transmitted intensity was amplified via a difference amplifier, and the amplified signal was recorded with a digital oscilloscope. To examine the retarded response of the optical transmission of the magnetic fluid film as the external magnetic field was switched on/off, the magnetic field was also monitored by measuring the current that flowed through the solenoids. This was done by connecting a resistor in series between the current source and the solenoid and by monitoring the voltage across the resistor. In order to detect the retarding time, defined as the time interval between the switching-on/off of the external field and the variation of the transmission through the film, the

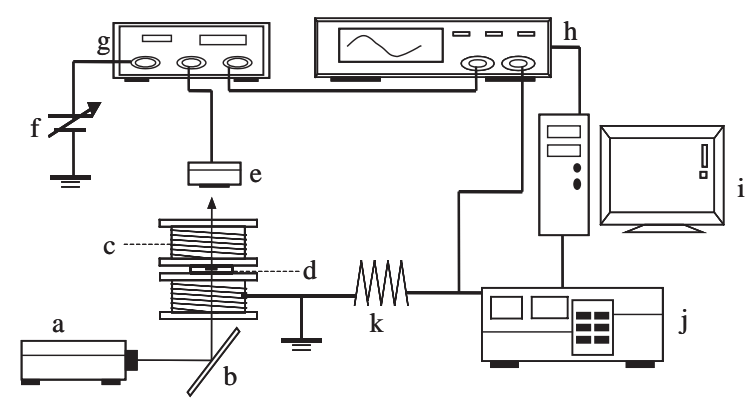

Fig. 1. Experimental setup for observing the time-dependent intensity of the transmitted light through the magnetic fluid film under switching-on/off a external magnetic field. The instruments labeled are: (a) He-Ni laser $(\lambda=632.8 \mathrm{~nm})$, (b) mirror, (c) solenoid, (d) magnetic fluid film, (e) high speed photodetector, (f) DC voltage source, (g) differential amplifier (gain =11), (h) oscilloscope, (i) PC, (j) current source, and (k) resistance. 
signals from the resistor and the photo-detector were synchronized first for each measurement. In this work, the time resolution used in the synchronization was pico-seconds.

\section{Results and discussion}

Firstly, the response of the optical transmission of magnetic fluid films under the switching-on of the external magnetic field (referred as the charging process) was investigated. Fig. 2(a) shows the time-dependent field strength. The field was turned on at $0.384 \mathrm{~s}\left(\equiv t_{\mathrm{H} 1}\right)$ to $120 \mathrm{Oe}$ and was kept at 120 Oe. The corresponding instant optical transmittance through the magnetic fluid film was detected and shown in Fig. 2(b). It is observed that the transmittance remains unchanged even when the external magnetic field is activated, i.e. at $0.384 \mathrm{~s}$, and starts to reduce at $0.408 \mathrm{~s}$ ( $\left.\equiv t_{\mathrm{T} 1}\right)$. Thus, the retarding time $r t_{1}$ of the transmittance response of the magnetic fluid film under the charging process to $120 \mathrm{Oe}$ is $24 \mathrm{~ms}$ via $r t_{1}=$

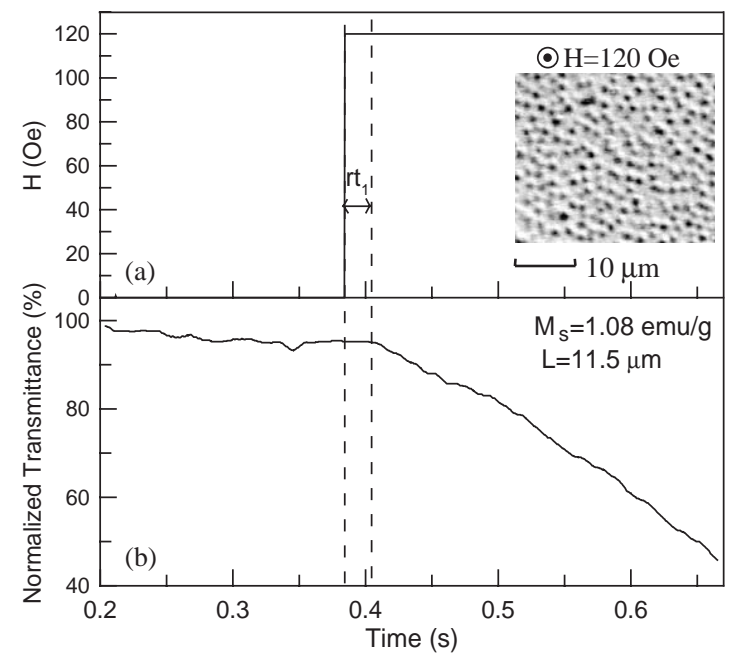

Fig. 2. Time-dependent (a) applied magnetic field $H$, (b) normalized optical transmittance through the magnetic fluid film during the charging process. The applied field strength shown here is $120 \mathrm{Oe}$, the concentration $M_{\mathrm{s}}$ and the thickness of the magnetic fluid film are $1.08 \mathrm{emu} / \mathrm{g}$ and $11.5 \mu \mathrm{m}$, respectively. The dashed lines show the retarding time (denoted by $r t_{1}$ ) of the transmittance response to the switch-on of the external field. The inset in (a) is the structural patterns of the magnetic clusters in the film under 120 Oe.
$t_{\mathrm{T} 1}-t_{\mathrm{H} 1}$. The transmittance through the magnetic fluid film then gradually decreased to a certain saturated value as time increased beyond $0.408 \mathrm{~s}$.

The reduction in the optical transmission through the magnetic fluid film with magnetic clusters shown in the inset of Fig. 2(a) may be attributed to the dichroism or the blocking effect of these clusters [13-16]. Since the formation of magnetic clusters is enhanced under a higher field, the $r t_{1}$ may change with the external field strength. To clarify this point, the retarding time $r t_{1}$ was measured as a function of the magnetic field strength for the charging process and was plotted in Fig. 3. It is clear that the retarding time $r t_{1}$ is decreased as the field strength is raised.

Furthermore, the real-time variation in the optical transmission after the switch-on of the external field is investigated. The result is shown in Fig. 4 for the field of $300 \mathrm{Oe}$. In the figure, one can see that the normalized transmittance $T_{\mathrm{N}}$ decays exponentially after switching on the field and follows $T_{\mathrm{N}}=$ $\mathrm{e}^{-\left(t-t_{0}\right) / \tau_{1}}$, where $t_{0}$ denotes the instance at which $T_{\mathrm{N}}$ starts to reduce. The $\tau_{1}$ is the time constant and is found to be $0.83 \mathrm{~s}$ in case. Since the reduction in the optical transmission is due to the formation of column clusters, the probed value of $\tau_{1}$ means that the formation rate of magnetic clusters is $1 / 0.83 \mathrm{~s}^{-1}$.

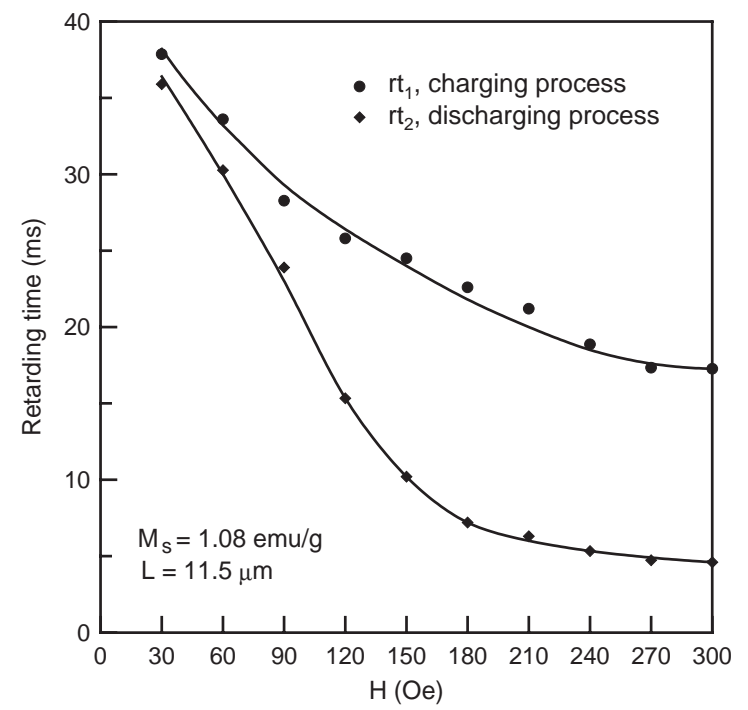

Fig. 3. Magnetic-field dependent retarding time of the magnetic fluid film for the charging and the discharging processes. 


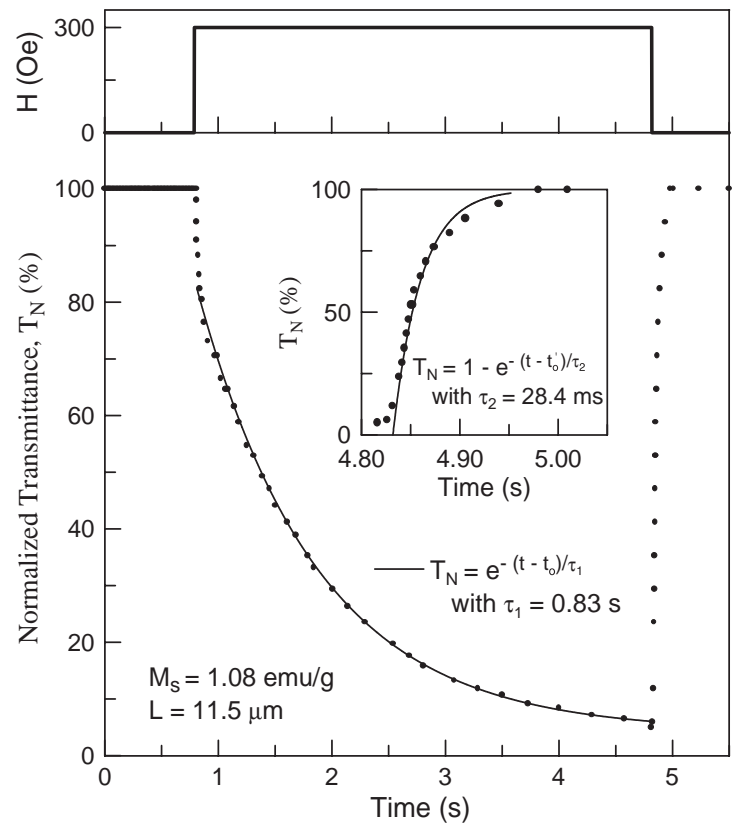

Fig. 4. Real-time response in the transmittance through the magnetic fluid film under the application of a step-like magnetic field with a magnitude of $300 \mathrm{Oe}$. The magnified plot for dynamic variation in the transmittance under the switch-off of the field is shown in the inset.

When the field was switched off (referred as the discharging process) from the 120 Oe at $1.593 \mathrm{~s}$ as shown in Fig. 5(a), a retarding phenomenon in the optical transmittance was observed. The transmittance did not increase until $1.608 \mathrm{~s}$, as shown in Fig. 5(b). This resulted in $15 \mathrm{~ms}$ for the retarding time $r t_{2}$ of the transmittance response for the discharging process from 120 Oe. The $r t_{2}-H$ curve also exhibits a similar trend as the $r t_{1}-H$ curve in Fig. 3 except a shift to the region with smaller values for retarding time.

The time-dependent transmittance through the film after switching-off the field is detected and shown in the inset of Fig. 4. We found that the normalized transmittance $T_{\mathrm{N}}$ became higher exponentially and can be described as $T_{\mathrm{N}}=1-$ $\mathrm{e}^{-\left(t-t_{0}^{\prime}\right) / \tau_{2}}$, where $t_{0}^{\prime}$ denotes the instance at which the transmittance starts to increase, and $\tau_{2}$ is the time constant of the cluster relaxation and found to be $28.4 \mathrm{~ms}$ in case. Thus, the magnetic clusters relax at the rate of $1 / 28.4 \mathrm{~ms}^{-1}$.

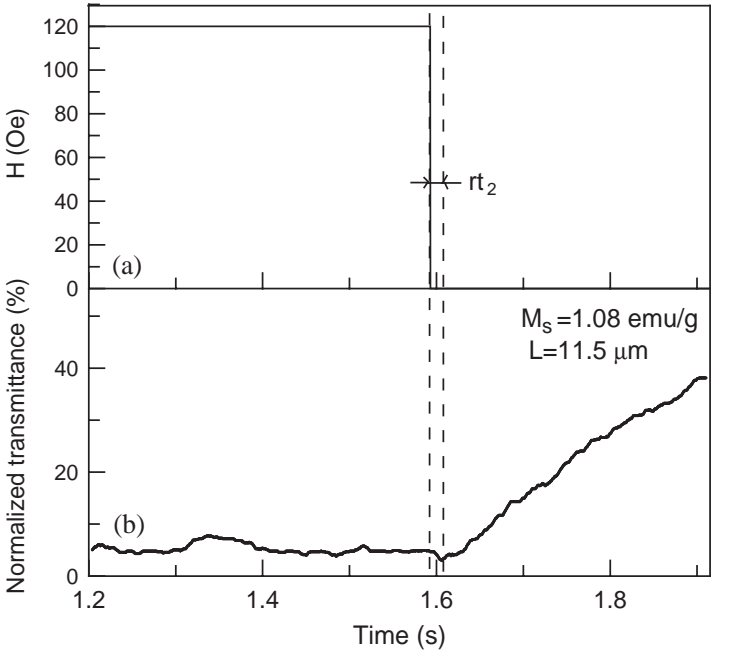

Fig. 5. Time dependencies of: (a) applied magnetic field $H$, (b) normalized optical transmittance through the magnetic fluid film during the discharging process. The applied field is switched off from $120 \mathrm{Oe}$, and the concentration $M_{\mathrm{s}}$ and the thickness of the magnetic fluid film are $1.08 \mathrm{emu} / \mathrm{g}$ and $11.5 \mu \mathrm{m}$, respectively. The dashed lines show the retarding time (denoted by $r t_{2}$ ) of the transmittance response to the switch-off of the external field.

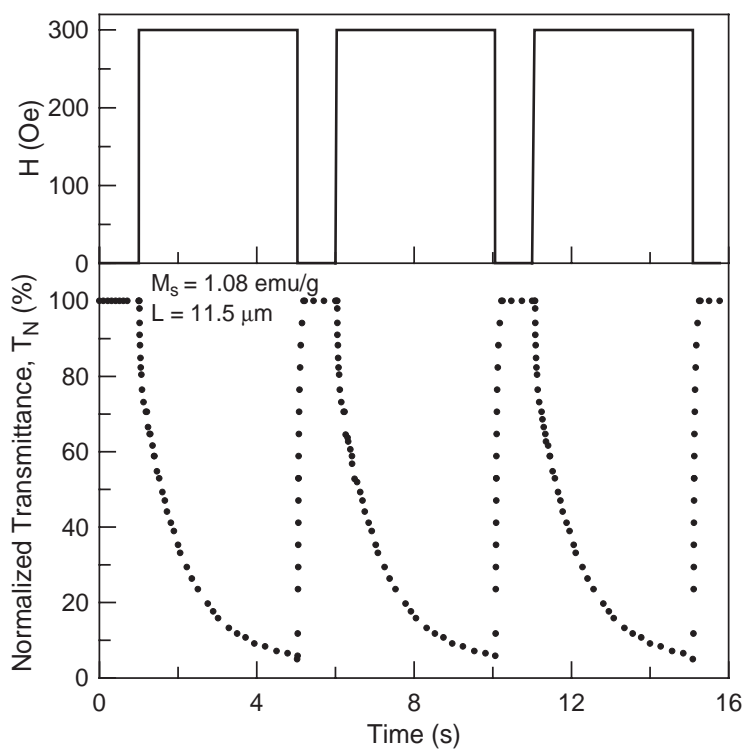

Fig. 6. (a) Periodically applied magnetic field and (b) the corresponding dynamic variation in the optical transmission through the magnetic fluid film. 
So far, we have discussed in detail the dynamic response of the optical transmission through the magnetic fluid film experiencing one circle of switching-on and -off the external magnetic field. We then investigate the time-dependent optical transmission of the magnetic fluid film under a periodically applied field. Fig. 6(a) plots the periodic field and the corresponding response in the transmission is shown in Fig. 6(b). A repeatable and periodic variation in the transmitted light was observed.

\section{Conclusion}

The optical transmission through a magnetic fluid film exhibits a retarded effect upon the switching-on of an external magnetic field, whose strength is high enough to cause the formation of magnetic clusters in the film. It was found that the retarding time could be manipulated from tens of milliseconds to several milliseconds by controlling the external field strength. After the retardation, the transmittance decays exponentially with a time constant of less than $1 \mathrm{~s}$. When the external field is switched off, the retarded variation in the transmission also occurs and the transmission follows an exponential behavior to grow. The dynamic variation in the transmission through the magnetic fluid film is repeatable under a periodically applied magnetic field.

\section{Acknowledgements}

This work is supported by National Science Council of ROC under Grant Nos. NSC92-2112M-003-010 and 92-2212-E-212-011.

\section{References}

[1] P.C. Scholten, J. Phys. D 13 (1980) L231.

[2] J.P. Llewelyn, J. Phys. D 16 (1983) 95.

[3] Yu.N. Skibln, V.V. Chekanov, Yu.L. Raikher, Sov. Phys. JETP 45 (1977) 496.

[4] R.W. Chantrell, A. Bradbury, S. Menear, J. Appl. Phys. 57 (1985) 4268.

[5] P. Goldberg, J. Hansford, P.J. van Heerden, J. Appl. Phys. 42 (1971) 3874.

[6] P.A. Martinet, Rheol. Acta 13 (1974) 260.

[7] W.E. Haas, J.E. Adams, Appl. Phys. Lett. 27 (1975) 57.

[8] P.C. Scholten, IEEE Trans. Magn. MAG-11 (1975) 1400.

[9] H.W. Davies, J.P. Llewellyn, J. Phys. D 12 (1979) 1357.

[10] J.-C. Bacri, R. Perzynski, D. Salin, J. Servais, J. Phys. 48 (1987) 1385

[11] S. Taketomi, S. Ogawa, H. Miyajima, S. Chikazumi, K. Nakao, T. Sakakibara, T. Goto, N. Miura, J. Appl. Phys. 64 (1988) 5846.

[12] N.A. Yusuf, Jpn. J. Appl. Phys. 27 (1988) 2418.

[13] S. Taketomi, Jpn. J. Appl. Phys. 22 (1983) 1137.

[14] S. Taketomi, M. Ukita, M. Mizukami, H. Miyajima, S. Chikazumi, J. Phys. Soc. Jpn. 56 (1987) 3362.

[15] C.-Y. Hong, J. Appl. Phys. 85 (1999) 5962.

[16] S.Y. Yang, Y.P. Chiu, H.E. Horng, C.-Y. Hong, B.Y. Jeang, H.C. Yang, Appl. Phys. Lett. 79 (2001) 2372. 